group. The SPARCC scores in TNF-i group also decreased significantly (Figure1, $P<0.01$ ). There was no significant progress in fat metaplaisa, bone erosions, sclerosis and ankylosis during the follow-up period $(P>0.05)$. Even though the inflammatory indexes and clinical evaluation of non-TNF-i group did not improved remarkably, SPARCC score were significantly reduced at 4-6 months and 1-2 years $(P<0.05)$.

Conclusions: TNF-i could reduce clinical and imaging inflammatory degree. Prolonged the interval of TNF-i treatment could maintain low disease activity and improve bone marrow edema, whereas fat metaplasia, bone erosion, sclerosis and ankylosis were not exacerbated.

Disclosure of Interest: None declared

DOI: 10.1136/annrheumdis-2017-eular.4775

\section{THU0400 RISK FACTORS OF SAGITTAL TRANSLATION AFTER PEDICLE SUBTRACTION OSTEOTOMY ON ANKYLOSING SPONDYLITIS}

Y.-S. Park, J. Kim, J.-S. Park. Department of Orthopaedic Surgery, Hanyang University Guri Hospital, Guri city, Korea, Republic Of

Background: Few studies on sagittal translation and its risk factors after pedicle subtraction osteotomy (PSO) in ankylosing spondylitis (AS) patients have been conducted. There is also no study on overall evaluation of radiologic parameters as the candidate of its risk factor.

Objectives: The aim of this study was to report the cases of sagittal translation which developed after PSO in AS patients with kyphotic deformity and to analyze its risk factors

Methods: The subjects of this study were 53 AS patients (58 cases) who underwent PSO to correct their kyphotic deformity between March 2006 and August 2016. The 53 subjects consisted of 45 males and 8 females. Their mean age was 39.312 Tit'>7.9 (range: 29-67). After osteotomy, the patient was examined for the presence of sagittal translation in the correction site through intraoperative radiograph. The low modified Stoke AS spine score (mSASSS) was measured before the surgery. The vertebral parameters such as lumbar lordosis angle, thoracic kyphotic angle, and sagittal vertical axis, and the pelvic parameters such as pelvic incidence, pelvic tilt, and sacral slope were also measured before and after the surgery.

The subjects were grouped according to the presence and absence of sagittal translation, and their radiologic parameters were compared. In addition, the correlation between sagittal translation and each parameter was analyzed. Complications that developed during and after the surgery were also analyzed. Results: Sagittal translation developed in 16 subjects (30\%) or 17 cases $(29.3 \%)$. The mean lumbar lordosis angle and the mean sagittal vertical axis of both the sagittal translation (ST) group and the non-sagittal translation (Non-ST) group were successfully corrected ( $\mathrm{p}=0.000$, respectively). A significant difference in preoperative mean sacral slope was observed between the groups $(p=0.045)$. The ST group showed a significantly higher mSASSS $(48.1 \pm 20.7)$ than the Non-ST group (36.8 \pm 16.2$)(p=0.002)$. In the multivariate regression analysis, sagittal translation was positively correlated with mSASSS (odds ratio $1.34, P=0.002$ ) and the preoperative sacral slope (odds ratio $1.46, P=0.009$ ), and negatively correlated with the difference between preoperative and postoperative thoracic kyphotic angle (odds ratio $0.68, P=0.01$ ). Both groups showed no finding of permanent neurologic complication after the surgery.

Conclusions: The incidence of sagittal translation after pedicle subtraction osteotomy was closely related with the severity of ankyloses in AS patients. Therefore, when pedicle subtraction osteotomy is performed for AS patients with severe ankyloses and high sacral slope, it is required that surgeon consider sagittal translation which could induce neurologic complication.

Disclosure of Interest: None declared

DOI: 10.1136/annrheumdis-2017-eular.4239

\section{THURSDAY, 15 JUNE 2017}

\section{Crystal diseases, metabolic bone diseases and bone diseases other than osteoporosis}

\section{THU0401 ULTRASOUND EVALUATION IN FOLLOW-UP OF URATE-LOWERING THERAPY IN GOUTY PATIENTS: THE USEFUL STUDY}

M. Forien ${ }^{1}$, E. Norkuviene ${ }^{2}$, P. Richette ${ }^{3}$, G. Mouterde ${ }^{4}$, H.-K. Ea ${ }^{3}$, F. Lioté ${ }^{3}$ M. Petraitis ${ }^{2}$, T. Bardin ${ }^{3}$, J. Ora ${ }^{3}$, P. Dieudé ${ }^{1}$, S. Ottaviani ${ }^{1} .{ }^{1}$ Rheumatology, Hôpital Bichat, Paris, France; ${ }^{2}$ Rheumatology, Lithuanian University of Health Sciences, Kaunas, Lithuania; ${ }^{3}$ Rheumatology, Lariboisière Hospital, Paris; ${ }^{4}$ Rheumatology, Lapeyronie Hospital, Montpellier, France

Background: Ultrasonography (US) has demonstrated its ability to detect urate deposition in gouty patients. Some US features have been suggested to be specific such as tophus and the double contour (DC) sign. In contrast to the usefulness of US for diagnosis, data are lacking on its role in follow-up of gout deposition after initiation of urate-lowering therapy (ULT).

Objectives: We aimed to determine the ability of US to show disappearance of urate deposits in gouty patients requiring ULT.
Methods: We performed a 6-month multicentre prospective study. To be included in the study, patients needed to have: i) a proven gout (identification of monosodic urate crystal in synovial fluid analysis or tophus aspiration), ii) presence of US features of gout (tophus and/or DC sign) at knee and/or first metatarsophalangeal joints (MTP1s). Serum uric-acid (SUA) level was assessed at baseline, M3 and M6. US evaluations were performed at baseline, M3 and M6 after starting ULT, by one local rheumatologist, blinded to SUA levels and clinical data. The primary outcome was the decrease (absolute value and percentage of decrease) of US tophus after 6 months of ULT, according to the final SUA levels. The secondary outcome was the mean percentage of joint sites with DC sign disappearance. Three stages of SUA levels were defined (high SUA levels: $>360 \mu \mathrm{mol} / \mathrm{l}$, low SUA: $300-360 \mu \mathrm{mol} / \mathrm{l}$, very low SUA: $<300 \mu \mathrm{mol} / \mathrm{l}$.

Results: A total of 79 gouty patients (mean \pm SD age $61.8 \pm 14$ years, $91 \%$ of males) were included. The mean disease duration was $6.3 \pm 6.1$ years. Tophi were found at clinical exam in $29 \%$ of patients. Baseline SUA levels were $530 \pm 97$ $\mu \mathrm{mol} / \mathrm{l}$. At least one US tophus and DC sign were found in $74(93.7 \%)$ and 68 $(86.1 \%)$ of patients, respectively. Allopurinol and febuxostat was started in 26 $(33 \%)$ and $53(67 \%)$ patients, respectively. A total of 67 patients were completers at 6 months. Among those M6 completers, 39 and 18 patients achieved a very low and low SUA levels, respectively. The 10 remaining patients maintained high SUA levels. Comparison of US features of gout modifications between the 3 groups of final SUA levels revealed a higher decrease of US tophus size and higher proportion of DC sign dissolution among patients with lowest SUA levels (Table 1). Additionally, final M6 SUA levels was associated with: decrease size of tophus $(r=0.5093[0.3012 ; 0.6711], P<0.0001)$, percentage of decrease of the tophus size $(r=0.5352$ [0.3332; 0.6902$], P<0.0001)$ and inversely correlated with the proportion of DC sign dissolution ( $r=-0.624[-0.763 ;-0.4298])$.

Table 1. Modifications of US features of gout after 6 months of ULT

\begin{tabular}{lcccc}
\hline M6 SUA levels, mmol/l & SUA $<300$ & SUA 300-360 & SUA $>360$ & $P^{*}$ \\
& $\mathrm{~N}=39$ & $\mathrm{~N}=18$ & $\mathrm{~N}=10$ & \\
\hline Delta size tophus, mean \pm SD mm & $-6.5 \pm 4.1$ & $-4.4 \pm 3.0$ & $-0.0 \pm 1.5$ & $\mathrm{P}=0.00046$ \\
\% of decrease of tophus & $-56.6 \pm 32.9$ & $-31.5 \pm 24.3$ & $-10.3 \pm 17.2$ & $\mathrm{P}=0.00028$ \\
\% of joint site with DC sign dissolution & 80.8 & 59.9 & 1.1 & $\mathrm{P}<0.0001$ \\
\hline
\end{tabular}

${ }^{*}$ Kruskal-Wallis test. DC: double contour; SD: standard deviation; SUA: serum uric acid levels.

Conclusions: US is able to detect decrease or disappearance of US urate deposits after ULT. Additionally, the decrease of US deposits is strongly correlated with lowest SUA levels. These data suggest that US could be useful for ULT management in gouty patients.

Disclosure of Interest: None declared

DOI: 10.1136/annrheumdis-2017-eular.4941

\section{THU0402 ULTRASONOGRAPHY AND DUAL-ENERGY CT (DECT) DO NOT PROVIDE THE SAME QUANTIFICATION OF URATE DEPOSITION IN GOUT: RESULTS FROM A CROSS-SECTIONAL STUDY}

T. Pascart $^{1}$, A. Grandjean ${ }^{2}$, L. Norberciak ${ }^{3}$, V. Ducoulombier ${ }^{2}$, M. Motte $^{2}$ H. Luraschi ${ }^{2}$, M. Vandecandelaere ${ }^{2}$, C. Godart ${ }^{2}$, E. Houvenagel ${ }^{2}$, N. Namane $^{2}$ J.-F. Budzik ${ }^{4}$. ${ }^{1}$ Rheumatology; ${ }^{2}$ Hopital Saint Philibert, LOMME, France; ${ }^{3}$ Biostatistics; ${ }^{4}$ Radiology, Hopital Saint Philibert, LOMME, France

Background: Gout is due to monosodium urate (MSU) deposition in joints and soft tissues. Ultrasonography (US) and dual-energy CT (DECT) have been shown to be effective in detecting MSU deposits. Both techniques can examine tophi size. DECT is effective to identify soft-tissue MSU deposits and US can show joint deposition with the double contour (DC) sign. It is unknown if these two techniques provide the same quantification of the extent of urate deposition on a given patient.

Objectives: The main objective of this study is to compare the tophus size measured by US and by DECT. The secondary objective is to evaluate the correlation between the prevalence of the US DC sign and the global volume of urate deposits measured by DECT.

Methods: This prospective cross-sectional study included patients fulfilling the 2015 ACR/EUALR criteria for gout. Patients underwent US and DECT examinations of their knees and feet. The largest US tophi was selected as the index tophus. US examination of the DC sign was performed on the femoropatellar joints, talo-crural joints and 1st metatarsophalangeal joints. Total volume of urate deposits of knees and feet was measured by DECT. The primary endpoint was the intra-class correlation coefficient (ICC) of the volume of the index tophus measured by US and DECT [Cl 95\%].

Results: A total of 64 patients were included in the study, of which 35 patients presented with at least one US tophus. Patients were in average $64.5 \pm 16.3$ years old, $84.4 \%$ were male, had an average ACR/EULAR score of $13.6 \pm 2.5$, and disease duration was $12 \pm 14.7$ years. Overall, 44 patients $(68.8 \%)$ were currently taking urate lowering therapy and 22 patients $(34.4 \%)$ had clinical tophi. Out of the 35 US selected largest tophi, 6 tophi were not seen in DECT. Of the tophi identified with both techniques, 21 were localized in the feet and 8 in the knees. The ICC of the tophus volume assessment by US and DECT was 0.45 [0.12-0.69]. The average volume of the largest US tophi was $2.7 \pm 6.5 \mathrm{~cm}^{3}$ and $1.5 \pm 3.3 \mathrm{~cm}^{3}$ when measured by DECT. If the index tophus was localized in the knee, the ICC was $0.36[0-0.82]$ and was $0.68[0.37-0.86]$ if the tophus was in 
the foot. The Spearman correlation coefficient between the DECT urate volume and the number of joints with a positive DC sign was 0.15 .

Conclusions: US and DECT do not provide the same assessment of tophus size. The correlation is improved when considering tophi localized in the feet. The number of joints with positive DC sign does not correlate to the volume of urate deposition in the soft tissues measured by DECT.

Disclosure of Interest: None declared

DOI: 10.1136/annrheumdis-2017-eular.4118

\section{THU0403 URATE-LOWERING TREATMENT AND RISK OF INCIDENT UROLITHIASIS IN PEOPLE WITH GOUT: A NESTED CASE-CONTROL STUDY}

C.-F. Kuo, J.-S. Chen. Division of Rheumatology, Allergy and Immunology, Chang Gung Memorial Hospital, Taoyuan, Taiwan, Province of China

Background: A higher risk of urolithiasis has been reported in gout patients. However, whether urate-lowering treatments, including both xanthine oxidase inhibotors and uricosuric agents, are beneficial to reduce the risk or urolithiasis in gout patients has not been examined.

Objectives: To investigate the independent associations between urate-lowering treatment (ULT) and the risk of urolithiasis in incident gout patients.

Methods: We conducted a nested case-control study based on the Taiwan National Health Insurance Research Database (NHIRD), which was used to identify 473,858 newly diagnosis gout patients during the period from January 1 , 2000 through December 31, 2004. All these patients were followed until December 31,2013 . We considered patients who first diagnose incident urolithiasis after the date of entry cohort (gout onset) as cases and the diagnostic date was defined as index date. Each case was matched up to five eligible controls whose follow-up period included the case's index date by sex, birth of year and gout diagnosis year. And the index date of case was assigned to the matched controls. Odds ratios (ORs) and $95 \%$ confidence interval $(\mathrm{Cl})$ of urolithiasis associated with cumulative defined daily dose (cDDD) of xanthine oxidase inhibitor and uricosuric agents were main ourcome measures.

Results: Gout patients with incident urolithiasis $(n=32,654)$ occurring after the initial diagnosis of gout aged 20-79 were age- and sex-matched 1:5 to 163,270 gout patients without urolithiasis. After adjusting for age, sex, urbanization status, income, occupation, and pertinent drugs and comorbidities, the OR of urolithiasis associated with use of ULT among gout patients were $1.04(95 \% \mathrm{Cl} 1.00$ to 1.07$)$ for those with 28-90 cDDD, 0.95 (95\% confidence interval 0.91-0.99) for 91-365 cDDD and 0.77 (95\% confidence interval $0.73-0.82)$ for $>365$ cDDD, compared with those with a cDDD $<28$. The OR $(95 \% \mathrm{Cl})$ for urolithiasis associated with xanthine oxidase inhibitor use was $0.94(0.89-0.99)$ for 28-90 cDDD, 0.90 $(0.84-0.97)$ for $91-365 \mathrm{cDDD}$ and $0.73(0.63-0.83)$ for $>365 \mathrm{cDDD}$. For uriscouric agents, the OR $(95 \% \mathrm{Cl})$ for urolithiasis among those with 28-91, 91-365 and $<365$ cDDD were $1.04(1.01,1.08), 0.94(0.90-0.98)$ and $0.78(0.73-0.84)$, respectively.

Conclusions: Higher ULT consumption was associated with a lower risk of urolithiasis. Xanthine oxidase inhibitors associated with reduced urolithiasis risk consistently across the range of consumption studied but for uricosuric agents inadequate cumulative dose results in a higher risk despite the risk reduced gradually with a higher cumulative dose.

Disclosure of Interest: None declared

DOI: 10.1136/annrheumdis-2017-eular.2913

\section{THU0404 SELF-REPORTED SEVERITY OF GOUT IN A PRIMARY CARE SETTING AND ASSOCIATED FACTORS - RESULTS FROM A GOUT SURVEY IN PRIMARY CARE}

A.J. Landgren, M. Dehlin, U. Bergsten, V. Sigurdardottir, P. Drivelegka, T. Zverkova Sandström, L. Jacobsson. Department of Rheumatology and Inflammation Research, Institution of Medicine, Sahlgrenska Academy. University of Gothenburg., Gothenburg, Sweden

Background: Patients with gout are usually seen in primary care. There are numerous reports regarding severity of gout in cohort studies and patients seen in more specialized care, less is known about the spectrum of severity of the disease in primary care.

Objectives: To describe the pattern of self-reported disease severity of gout and predictors thereof in a primary care setting.

Methods: All patients above 18 with an ICD10-diagnosis of gout at a health care visit in primary care (Jan 2015 through Aug 2016) were identified from primary care within the Western Sweden Health Care Region (WSHCR). 941 patients were identified and included in the study. They were sent a questionnaire with questions regarding comorbidities, demographics, gout characteristics and Health Assessment questionnaire ( $\mathrm{HAQ})$ reflecting disability. Self-reported disease severity was assessed by a three-level Likert scale (mild, moderate, severe). Possibly associated factors were analyzed by bivariate logistic regression (binary outcome: mild vs. moderate/severe) models and factors that were statistically significant were further analyzed by multivariate analysis.

Results: The response rate was $54 \%$. Response rates were significantly lower in women overall and in men under the age of 50 . Covariates that were significantly associated with more severe self-reported severity of gout in the bivariate logistic regression models (Table 1) were: female sex, hyperlipidemia, higher number of previous attacks, attack during last month, HAQ. In the multivariate analysis objective measures of gout severity such as more than ten previous attacks and attack during last month were strongly associated with the patients grading of severity, in addition to presence of hyperlipidemia (Table 1).

Table 1. Characteristics of gout patients stratified by disease severity and logistic regression models

\begin{tabular}{|c|c|c|c|c|c|}
\hline Covariates & $\begin{array}{c}\text { Mild }^{*} \\
\mathrm{n}=272\end{array}$ & $\begin{array}{c}\text { Moderate }^{*} \\
n=130\end{array}$ & $\begin{array}{c}\text { Severe } \\
n=62\end{array}$ & $\begin{array}{l}\text { Univariate analyses }{ }^{* *} \\
\text { OR }(95 \% \mathrm{Cl}) \#\end{array}$ & $\begin{array}{c}\text { Multivariate analyses } \\
\text { (OR }(95 \% \mathrm{Cl}) \#\end{array}$ \\
\hline Age in years (mean) & 70.8 & 71.9 & 71.8 & $1.01(0.99-1.02)$ & $\mathrm{N} / \mathrm{A}$ \\
\hline Female sex $(\%)$ & 12.9 & 18.5 & 22.6 & $1.67(1.01-2.76)$ & $1.54(0.85-2.80)$ \\
\hline Education $\leq 9$ years $(\%)$ & 24.8 & 27.6 & 46.6 & $1.46(0.97-2.21)$ & $\mathrm{N} / \mathrm{A}$ \\
\hline Diabetes (\%) & 22.8 & 27.1 & 27.4 & $1.29(0.84-1.98)$ & N/A \\
\hline Kidney disease (\%) & 6.6 & 9.3 & 11.3 & $1.59(0.81-3.12)$ & $\mathrm{N} / \mathrm{A}$ \\
\hline BMI (mean) & 27.9 & 28.2 & 27.8 & $1.02(0.97-1.06)$ & $\mathrm{N} / \mathrm{A}$ \\
\hline Hyperlipidemia (\%) & 29.4 & 44.2 & 46.8 & $2.00(1.36-2.95)$ & $2.13(1.39-3.28)$ \\
\hline \multicolumn{6}{|l|}{ Duration of gout in years } \\
\hline \multicolumn{6}{|c|}{ Total number of attacks (\%): } \\
\hline 1 & 19.5 & 6.4 & 12.1 & ref & ref \\
\hline $2-5$ & 50.0 & 40.8 & 39.7 & $1.93(1.01-3.67)$ & $1.51(0.77-2.96)$ \\
\hline $6-10$ & 14.8 & 20.8 & 13.8 & $2.98(1.42-6.25)$ & $2.12(0.97-4.62)$ \\
\hline$>10$ & 15.6 & 32.0 & 34.5 & $5.00(2.48-10.09)$ & $3.15(1.49-6.65)$ \\
\hline $\begin{array}{l}\text { Attack during the last } \\
\text { month }(\%)\end{array}$ & 11.5 & 30.4 & 30.6 & $3.43(2.10-5.61)$ & $1.81(1.36-2.41)$ \\
\hline ULT⿻ $(\%)$ & 46.3 & 50.8 & 50.0 & $0.85(0.46-1.55)$ & N/A \\
\hline $\mathrm{HAQ}^{\S}$ (mean) & 0.24 & 0.29 & 0.51 & $1.56(1.09-2.24)$ & $1.20(0.80-1.81)$ \\
\hline Tophus (\%) & 14.3 & 21.3 & 4.8 & $1.17(0.69-1.98)$ & $\mathrm{N} / \mathrm{A}$ \\
\hline
\end{tabular}

${ }^{*}$ Self-reported disease severity. ${ }^{* \star}$ ORs reflects yes vs no for dichotomous covariates, per year for age and duration and per unit for HAQ. \#Moderate/severe vs mild, "Urate-lowering therapy. Health assessment questionnaire.

Conclusions: Over $40 \%$ of patients with gout in a primary care setting rated their disease as moderate or severe. The validity of the patients rating was supported by other covariates reflecting disease severity. The observations that women and those with hyperlipidemia reported a more severe disease needs to be further explored.

Disclosure of Interest: None declared

DOI: 10.1136/annrheumdis-2017-eular.3452

\section{THU0405 KILLING TWO BIRDS WITH ONE STONE? THE DASH DIET AND THE RISK OF GOUT: 26-YEAR FOLLOW-UP OF A PROSPECTIVE COHORT}

S.K. Rai ${ }^{1}$, T.T. Fung ${ }^{2,3}$, N. Lu ${ }^{1}$, S.F. Keller ${ }^{1}$, G.C. Curhan ${ }^{4,5}$, H.K. Choi ${ }^{1,4}$ ${ }^{1}$ Division of Rheumatology, Allergy, and Immunology, Massachusetts General Hospital; ${ }^{2}$ Department of Nutrition, Harvard T.H. Chan School of Public Health; ${ }^{3}$ Department of Nutrition, Simmons College; ${ }^{4}$ Channing Division of Network Medicine, Department of Medicine, ${ }^{5}$ Renal Division, Department of Medicine, Brigham and Women's Hospital, Boston, United States

Background: The Dietary Approaches to Stop Hypertension (DASH) diet, which reduces blood pressure and is recommended in cardiovascular disease (CVD) $(1,2)$, has also been found to lower serum uric acid (SUA) levels, particularly among those with hyperuricemia (3). Thus, the DASH diet may be particularly useful in gout care by reducing both SUA and CVD risk, especially among patients with hypertension which affects $74 \%$ of gout patients (4), thereby "killing two birds with one stone". However, corresponding data for the risk of gout are not available. In contrast, a Western dietary pattern may increase the risk of gout.

Objectives: To prospectively examine the relation between the DASH and Western dietary patterns and the risk of gout among men.

Methods: Using the Health Professionals Follow-up Study, we prospectively examined the relation between the DASH and Western diets and incident gout in 44,444 male participants with no history of gout at baseline. Using validated food frequency questionnaires, each participant was assigned a DASH score (reflecting high intake of fruits, vegetables, nuts and legumes, low-fat dairy products, and whole grains, and low intake of sodium, sweetened beverages, and red and processed meats) and a Western pattern score (reflecting higher intake of red and processed meats, French fries, refined grains, sweets, and desserts). We identified incident cases of gout meeting the preliminary ACR survey criteria for gout, adjusting for potential confounders including age, total energy intake, body mass index (BMI), diuretic use, history of hypertension, history of renal failure, and intake of alcohol and coffee. We conducted stratified analyses to evaluate whether the association between the DASH and Western pattern scores and the risk of gout varied according to $\mathrm{BMI}$, alcohol use, and hypertension status.

Results: During 26 years of follow-up, we documented 1,731 confirmed cases of incident gout. A higher DASH score was associated with a lower risk for gout (multivariable relative risk [RR] for extreme quintiles, 0.68 [95\% confidence interval, 0.57 to 0.80 ]; $P$ for trend $<0.001$ ) (Table 1 ). In contrast, a higher Western pattern score was associated with an increased risk for gout (multivariable RR for extreme quintiles, 1.42 [95\% confidence interval, 1.16 to 1.74 ]; $P$ for trend $=0.005$ ) (Table 1). These associations persisted regardless of $\mathrm{BMI}$, alcohol use, and hypertension status, and there was no significant interaction with these variables (all $\mathrm{P}$ for interaction $>0.17$ ) 